\title{
Optimising experimental design for MEG resting state functional connectivity measurement
}

Lucrezia Liuzzi $^{1}$, Lauren E. Gascoyne ${ }^{1}$, Prejaas K. Tewarie ${ }^{1}$, Eleanor L. Barratt ${ }^{1}$, Elena Boto ${ }^{1}$ and Matthew J. Brookes ${ }^{1 *}$

${ }^{1}$ Sir Peter Mansfield Imaging Centre, School of Physics and Astronomy, University of Nottingham, University Park, Nottingham, UK.

\section{*Correspondence to:}

Dr M. J. Brookes,

Sir Peter Mansfield Imaging Centre,

School of Physics and Astronomy,

University of Nottingham,

University Park,

Nottingham

NG7 2RD

E-mail: matthew.brookes@nottingham.ac.uk

Pages: $\quad 27$

Words: $\quad 10,010$ (including references).

Figures: $\quad 7$

Running Title: Optimising experimental design for MEG resting state functional connectivity measurement

Keywords: Functional connectivity; Networks; Magnetoencephalography; MEG; Resting State; beamformer 


\section{ABSTRACT}

The study of functional connectivity using magnetoencephalography (MEG) is an expanding area of neuroimaging, and adds an extra dimension to the more common assessments made using fMRI. The importance of such metrics is growing, with recent demonstrations of their utility in clinical research, however previous reports suggest that whilst group level resting state connectivity is robust, single session recordings lack repeatability. Such robustness is critical if MEG measures in individual subjects are to prove clinically valuable. In the present paper, we test how practical aspects of experimental design affect the intra-subject repeatability of MEG findings; specifically we assess the effect of coregistration method and data recording duration. We show that the use of a foam head-cast, which is known to improve coregistration accuracy, increased significantly the between session repeatability of both beamformer reconstruction and connectivity estimation. We also show that recording duration is a critical parameter, with large improvements in repeatability apparent when using ten minute, compared to five minute recordings. Further analyses suggest that the origin of this latter effect is not underpinned by technical aspects of source reconstruction, but rather by a genuine effect of brain state; short recordings are simply inefficient at capturing the canonical MEG network in a single subject. Our results provide important insights on experimental design and will prove valuable for future MEG connectivity studies. 


\section{INTRODUCTION}

The measurement of covariation between neuroimaging signals generated in spatially separate brain regions facilitates the elucidation and characterisation of brain networks (Beckmann et al., 2005; Corbetta et al., 1998; Fox et al., 2005; Fox and Raichle, 2007; Friston, 1994; Raichle et al., 2001; Smith et al., 2009). These networks, and the functional connectivities that define them, are thought to support core mental processes with some related to sensory processing and others associated with high level cognition. Because these networks can be observed even when subjects are notionally "at rest" (i.e. no specific task paradigm is used) they are often termed resting state networks (RSNs) (Beckmann et al., 2005). Although predominantly measured using functional magnetic resonance imaging (fMRI), a growing body of work shows that network characterisation via magnetoencephalography (MEG) offers novel insights into the neural mechanisms that underlie functional connectivity (Hall et al., 2014; O'Neill et al., 2015a; Schölvinck et al., 2013). MEG involves assessment of the magnetic fields that are induced outside the head by neural current flow in the brain. Mathematical modelling of these fields subsequently allows construction of 3D images showing moment to moment changes in brain current. Because MEG signals are generated directly by neurons, the electrophysiological basis of connectivity can be probed. Furthermore, the excellent temporal precision afforded by MEG allows estimation of dynamic changes in network structure (Baker et al., 2014; Brookes et al., 2014; O'Neill et al., 2015a). The importance of characterising electrophysiological connectivity is growing, with numerous demonstrations that connections are perturbed in pathologies (Brookes et al., 2016; Friston, 1998; Guggisberg et al., 2008; Kessler et al., 2014; Palaniyappan and Liddle, 2012; Schnitzler and Gross, 2005; Stufflebeam et al., 2011; Tewarie et al., 2014; van Dellen et al., 2012). However, demonstrations have typically been made on large subject groups, with little attention paid to the accuracy of measurements within individual subjects. Such accuracy is critical if MEG connectivity is to provide diagnostic and clinically relevant information.

To date, most studies that have examined the robustness of MEG connectivity estimation suggest relatively poor reproducibility. For example, Wens et al. (2014) showed that whilst group level connectivity within several well-known networks is stable, there is significant variability at the individual subject level. Colclough et al. (2016) tested the between session repeatability of a large number of functional connectivity measurements, showing that although group level inference is reliable, network metrics vary across individuals. The authors also showed that even separate measures in a single subject lack reproducibility. Tewarie et al. (2016) used MEG to predict the canonical RSNs observed in fMRI; whilst predictions were robust at the group level, they fared less well within individuals and no significant inter-individual differences in connectivity were predicted. 
Similarly, O'Neill et al. (2016) showed that dynamic measures of functional connectivity are also variable across subjects. Although the reason for this poor reliability is not well understood, it likely results from a combination of genuine differences (Finn et al., 2015) (i.e. even within a single subject, differences in brain state between two experimental recordings could generate marked differences in network connectivity) and instrumental imprecision. Regarding the latter, MEG has relatively low signal to noise ratio (SNR) since environmental and biomagnetic fields of no interest (e.g. from lab equipment, heart and muscles) are larger than neuromagnetic fields. In addition, source localisation (reconstructing images of current density using extracranial magnetic fields) is based upon an ill-posed problem which reduces spatial resolution. Another significant limitation is coregistration error. The accuracy of source localisation is dependent not only on data quality, but also on precise forward models (models of magnetic fields that would be observed at the sensors given a known current distribution). Forward models, in turn, require accurate knowledge of the location and orientation of MEG sensors relative to brain anatomy. Importantly (and perhaps counterintuitively) as SNR is increased, the requirement for high spatial precision also increases. Unfortunately at present, coregistration usually relies on digitisation and surface matching procedures which can cause errors of several millimetres that propagate into source reconstructions. If the potential of MEG to characterise network connectivity in individual subjects is to be realised, then these sources of error must be minimised.

Here, using simulations and experiments, we probe how test-retest repeatability of functional connectivity in MEG can be optimised by changing practical aspects of experimental design. We concentrate on two parameters; recording duration and coregistration method. It is clear that longer recordings will maximise SNR, and previous work (Brookes et al., 2010, 2008) shows that source reconstruction accuracy and spatial resolution are improved as recording duration (or bandwidth) is increased. Here, using simulated and experimental MEG data, we investigate the effect that changing recording duration has on network connectivity. Investigating the effect of coregistration error in real MEG data is challenging since such errors are inherent to all experimental measures and no ground truth is known. Here, we address this issue by adopting a recently developed approach, employing foam head-casts (constructed using 3D printing) (Troebinger et al., 2014). The internal surface of these head-casts fits to the subject's scalp, whilst the external surface fits the MEG scanner helmet. In this way we are able to lock the head in place, minimising motion and coregistration error. In what follows, we will show that recording duration and coregistration have a marked effect on the repeatability of source localisation, and functional connectivity. Our results will provide advice for the design of future scanning protocols, particularly in studies of individual subjects. 


\section{SIMULATIONS}

In order to examine the effect of coregistration error and recording duration on connectivity estimation, we first undertook a set of simulations. All simulations were based upon the geometry of a CTF 275 channel axial gradiometer system ( $5 \mathrm{~cm}$ baseline) operating in third order gradiometer configuration. Brain anatomy, head shape, and system geometry were based upon a real experimental recording session. In everything that follows, the simulated sampling frequency was $600 \mathrm{~Hz}$; all simulated sources were assumed to be dipolar; the forward field was computed using a multiple local sphere head model and the formula derived by Sarvas (1987). The locations of all simulated sources were set according to the centre of mass of 78 anatomically defined cortical regions, extracted from the automated anatomical labelling $(A A L)$ atlas.

\section{Simulation Methods}

\section{Simulated data}

Two dipoles, henceforth known as the seed and test dipole, were simulated in homologous regions of somatosensory cortex, with locations and orientations represented by $\boldsymbol{\theta}_{\text {seed }}$ and $\boldsymbol{\theta}_{\text {test }}$ respectively. (I.e. $\boldsymbol{\theta}$ represents both location (set according to the AAL regions) and orientation, which was confined to the tangential plane and set at a random angle with respect to the azimuthal direction). The dipole timecourses, denoted $q_{\text {seed }}(t)$ and $q_{\text {test }}(t)$, were simulated in order to mimic genuine functional connectivity between regions. Mathematically,

$q_{\text {seed }}(t)=b(t)+e_{1}(t)$,

and

$q_{\text {test }}(t)=b(t+\tau)+e_{2}(t)$.

Here, $b(t)$ was generated by frequency filtering Gaussian noise into the beta band (13-30 $\mathrm{Hz}$ ). This was added to $q_{\text {seed }}(t)$ and a time/phase shifted version was added to $q_{\text {test }}(t)$ in order to simulate functional connectivity between locations. In addition, $e_{1}(t)$ and $e_{2}(t)$, which represent two orthogonal beta band filtered Gaussian noise timecourses, with amplitude equal to $30 \%$ of $b(t)$, were added. In this way, the simulated timecourses represent regional brain signals which exhibit partial connectivity. Note that $\tau=10 \mathrm{~ms}$. To make the simulation more realistic, in addition to the seed and test dipoles, we simulated a further 76 dipoles located at the centres of mass of the remaining AAL regions. These "dipoles of no interest" had amplitude equal to $5 \%$ of the seed and test signals. The final dataset was simulated as

$\boldsymbol{B}(t)=a_{\text {seed }} \boldsymbol{l}\left(\boldsymbol{\theta}_{\text {seed }}\right) q_{\text {seed }}(t)+a_{\text {test }} \boldsymbol{l}\left(\boldsymbol{\theta}_{\text {test }}\right) q_{\text {test }}(t)+\left(\sum_{i=1}^{76} a_{i} \boldsymbol{l}\left(\boldsymbol{\theta}_{\boldsymbol{i}}\right) q_{i}(t)\right)+\boldsymbol{E}(t)$.

Here, $a_{\text {seed }}$ and $a_{\text {test }}$ represent the amplitudes of the seed and test dipoles respectively. $\boldsymbol{l}(\boldsymbol{\theta})$ represents the forward field for location/orientation $\boldsymbol{\theta}$. The summation represents the contribution of the dipoles of no interest and $\boldsymbol{E}(t)$ represents sensor level interference. 
Notice that $\boldsymbol{B}(t), \boldsymbol{E}(t)$ and $\boldsymbol{l}(\boldsymbol{\theta})$ are vectors of dimension $N_{\text {chans }} \times 1$, where $N_{\text {chans }}$ represents the number of MEG sensors. $\boldsymbol{E}(t)$ was generated using real MEG data from an empty room noise recording, acquired using a 275 channel CTF system in third order gradiometer configuration, and filtered into the beta band. The simulated SNR (defined as the ratio of the norms of the field from all dipoles and the interference field) was set to 1 .

\section{Beamformer reconstruction}

Following simulation of MEG data, reconstruction in source space was achieved via beamforming. Briefly, an estimate of electrical source strength, $\hat{q}(\boldsymbol{\theta}, t)$, at cortical location and orientation $\boldsymbol{\theta}$ was given by a weighted sum of sensor measurements so that:

$\hat{q}(\boldsymbol{\theta}, t)=\boldsymbol{w}^{T}(\boldsymbol{\theta}) \mathbf{B}(\mathrm{t})$

Note that the 'hat' notation represents an estimate; i.e. $\hat{q}$ is the reconstructed estimate of $q$ made using only the MEG data. $\boldsymbol{w}(\boldsymbol{\theta})$ is a vector of weighting parameters (dimension $N_{\text {chans }} \times 1$ ) tuned to location/orientation $\boldsymbol{\theta}$ and derived based on variance minimisation; the overall variance in $\hat{q}$ is minimised with the constraint that signals originating from $\boldsymbol{\theta}$ remain. Mathematically

$\mathbf{w}_{j}^{\mathrm{T}}=\frac{\hat{\boldsymbol{l}}^{\mathbf{T}}(\boldsymbol{\theta})\{\mathbf{C}\}^{-1}}{\hat{\boldsymbol{l}}^{\mathbf{T}}(\boldsymbol{\theta})\{\mathbf{C}\}^{-1} \hat{\boldsymbol{l}}(\boldsymbol{\theta})}$

where $\mathbf{C}=\left\langle\mathbf{B}(\mathbf{t})^{\mathbf{T}} \mathbf{B}(\mathbf{t})\right\rangle$ and is approximated by the data covariance matrix. The vector $\hat{\boldsymbol{l}}(\boldsymbol{\theta})$ represents the best estimate of the 'true' lead field, $\boldsymbol{l}(\boldsymbol{\theta})$. In the following simulations, this beamforming method was used to generate estimates, $\hat{q}_{\text {seed }}(t)$ and $\hat{q}_{\text {test }}(t)$.

\section{Simulating coregistration error}

Coregistration errors and subject movement during an MEG recording represent a stochastic error on the assumed locations of the MEG sensors with respect to the head. This error manifests in source reconstruction as a difference between the 'real' lead field, $\boldsymbol{l}(\boldsymbol{\theta})$, and the lead field used for beamformer reconstruction, $\hat{\boldsymbol{l}}(\boldsymbol{\theta})$. Here, we simulated coregistration error by adding a random translational and rotational error to the MEG sensor locations and orientations; this was achieved by a simple coordinate transform. The absolute value of translational and rotational error was fixed, while the proportions along or about each axis were randomised with values taken from a Gaussian distribution. In other words, for translational error, an absolute value was fixed at, say $1 \mathrm{~mm}$, but the direction of the error was random. Similarly, for rotational error, a value was fixed, say $1^{\circ}$ and the axes about which this rotation occurred (the contribution of pitch, yaw and roll) was random. (See Figure 1A.) Our simulation added translational and rotational error simultaneously and proportionally (i.e. $1 \mathrm{~mm} / 1^{\circ} ; 2 \mathrm{~mm} / 2^{\circ}$ and so on up to $15 \mathrm{~mm} / 15^{\circ}$ ). For each absolute error value, the simulation was repeated 100 times (different error directions/rotations each time) and results averaged. This whole process was repeated 10 
times with different realisations of simulated dipole timecourses. Results were averaged over iterations.

\section{Simulating data recording duration error}

Differences in recording duration were achieved by simulating different amounts of MEG data. Data duration was varied between $20 \mathrm{~s}$ and $60 \mathrm{~s}$ in steps of $10 \mathrm{~s}$, and between $60 \mathrm{~s}$ and $560 \mathrm{~s}$ in steps of $30 \mathrm{~s}$. For each duration, a MEG dataset was simulated 10 times and results averaged over iterations.

\section{MEG Connectivity metrics}

The temporal complexity of MEG signals means that a number of models to characterise functional connectivity have been proposed (Schölvinck et al., 2013). Most of these can be divided into two categories which exploit either phase or amplitude information. Phase methods (e.g. coherence, imaginary coherence, phase locking value or phase lag index) seek to measure a stable phase relationship between band limited signals measured at spatially separate locations. Amplitude methods (e.g. amplitude envelope correlation) seek to characterise temporal correlation between the envelopes of those same band limited signals. Here, we chose 5 separate connectivity metrics:

1. Amplitude envelope correlation (AEC): Reconstructed data, $\hat{q}_{\text {seed }}(t)$ and $\hat{q}_{\text {test }}(t)$ are frequency filtered into a band of interest and Hilbert transformed to derive the analytic signal. The absolute value of the analytic signal is then computed to generate the Hilbert envelope of band limited oscillations, denoted $\hat{E}_{\text {seed }}(t)$ and $\hat{E}_{\text {test }}(t)$. Pearson correlation, measured between $\hat{E}_{\text {seed }}(t)$ and $\hat{E}_{\text {test }}(t)$, then yields an estimate of amplitude envelope based functional connectivity.

2. Coherence (Coh) and Imaginary Coherence (ICoh): Beamformer reconstructed data, $\hat{q}_{\text {seed }}(t)$ and $\hat{q}_{\text {test }}(t)$ are again frequency filtered into a band of interest. Coherence is calculated as

$C_{q_{\text {seed }} q_{\text {test }}}(f)=\frac{s_{q_{\text {seed }} q_{\text {test }}(f)}}{\sqrt{S_{q_{\text {seed }} q_{\text {seed }}(f) s_{q_{\text {test }} q_{\text {test }}(f)}}}}$,

where $S_{x y}$ denotes the cross spectral density (if $x \neq y$ ) or auto spectral density (if $x=y)$. The result, $C_{q_{\text {seed }} q_{\text {test }}}(f)$, is a complex number, the magnitude of which is between 0 and 1 , with high values indicating the presence of a strong fixed phase relationship between $\hat{q}_{\text {seed }}(t)$ and $\hat{q}_{\text {test }}(t)$ at frequency $f$. (Note $f$ is limited to the range imposed by the frequency filter.) Imaginary Coherence is simply the imaginary part of $C_{q_{\text {seed }} q_{\text {test }}}(f)$, and is often employed to remove zero-phase-lag effects, which are usually a result of signal leakage (see below). 
3. Phase Locking Value (PLV) assesses the extent to which the phase difference between 2 signals changes over time; high PLV indicates a phase difference that remains constant (a fixed phase relationship). $\hat{q}_{\text {seed }}(t)$ and $\hat{q}_{\text {test }}(t)$ are frequency filtered and Hilbert transformed to generate the analytic signal $\left(\hat{A}_{\text {seed }}(t)\right.$ and $\left.\hat{A}_{\text {test }}(t)\right)$. Instantaneous phases of the two signals, $\hat{\phi}_{\text {seed }}(t)$ and $\hat{\phi}_{\text {test }}(t)$, are then extracted $\left(\operatorname{as} \hat{\phi}_{x}(t)=\operatorname{atan}\left(\operatorname{Im}\left[\hat{A}_{x}(t)\right] / \operatorname{Re}\left[\hat{A}_{x}(t)\right]\right)\right)$ and the phase difference generated so that $\Delta \hat{\phi}(t)=\hat{\phi}_{\text {seed }}(t)-\hat{\phi}_{\text {test }}(t)$. Phase locking value is defined as

$P L V=\left|\left\langle e^{i \Delta \widehat{\phi}(t)}\right\rangle\right|$.

Note that we estimated PLV as the average over 50\% overlapping $10 \mathrm{~s}$ long windows covering the whole simulated dataset. (Colclough et al., 2016)

4. Phase Lag Index (PLI) is related to PLV but eliminates the zero phase lag components. PLI is defined as

$P L I=\left|\left\langle\operatorname{sign}\left(\operatorname{Im}\left[e^{i \Delta \widehat{\phi}(t)}\right]\right)\right\rangle\right|$

Again, we estimated PLI as the average over 50\% overlapping $10 \mathrm{~s}$ long windows covering the whole experiment. (Colclough et al., 2016; Fraschini et al., 2016)

A major confound when measuring functional connectivity using MEG arises due to the non-uniqueness of the beamformer solution. Specifically, two beamformer derived timecourses (e.g. $\hat{q}_{\text {seed }}(t)$ and $\hat{q}_{\text {test }}(t)$ ) may exhibit significant correlation/coherence, purely due to 'signal leakage' between regions. This manifests as a zero-phase lag correlation between the seed and test timecourses and, without careful control, it artifactually inflates estimated connectivity between regions (Maldjian et al., 2014). Signal leakage has been well studied, and a number of methods, including PLI and ICoh, are robust to it since zerophase-lag effects are inherently removed. However, the other methods described above (AEC, Coh, PLV) remain susceptible to leakage and, prior to their calculation, a correction must be undertaken. A number of methods to apply such correction are available (Brookes et al., 2012; Hipp et al., 2012; O'Neill et al., 2015b; Wens et al., 2015) most of which employ orthogonalisation of beamformer projected signals to remove zero lag effects. For the present work, prior to calculation of AEC, Coh and PLV, we adopted two approaches: for our simulations, we used a simple pairwise orthogonalisation between $\hat{q}_{\text {seed }}(t)$ and $\hat{q}_{\text {test }}(t)$, similar to that described in Brookes et al. (2012) and Hipp et al. (2012). For the experimental work described later, we were interested in all-to-all connectivity between 78 cortical regions and therefore a multivariate orthogonalisation scheme, proposed by Colclough et al. (2015) was used. Here, signals from all regions of interest are symmetrically orthogonalised within a single computation. Briefly, the method involves two steps: first, a set of orthonormal time-courses, closest to the data matrix and for which there is a simple analytic solution, is found. Second, by iteratively adjusting the 
lengths and orientations of the corrected vectors, the solution is finessed until it is as close as possible to the uncorrected timecourses. The result is a new set of timecourses which contain the orthogonalised time series. For both the pairwise and multivariate approaches this leakage reduction step was applied after frequency filtering but prior to Coh calculation, and prior to Hilbert transformation for calculation of AEC and PLV.

\section{Simulation Results}

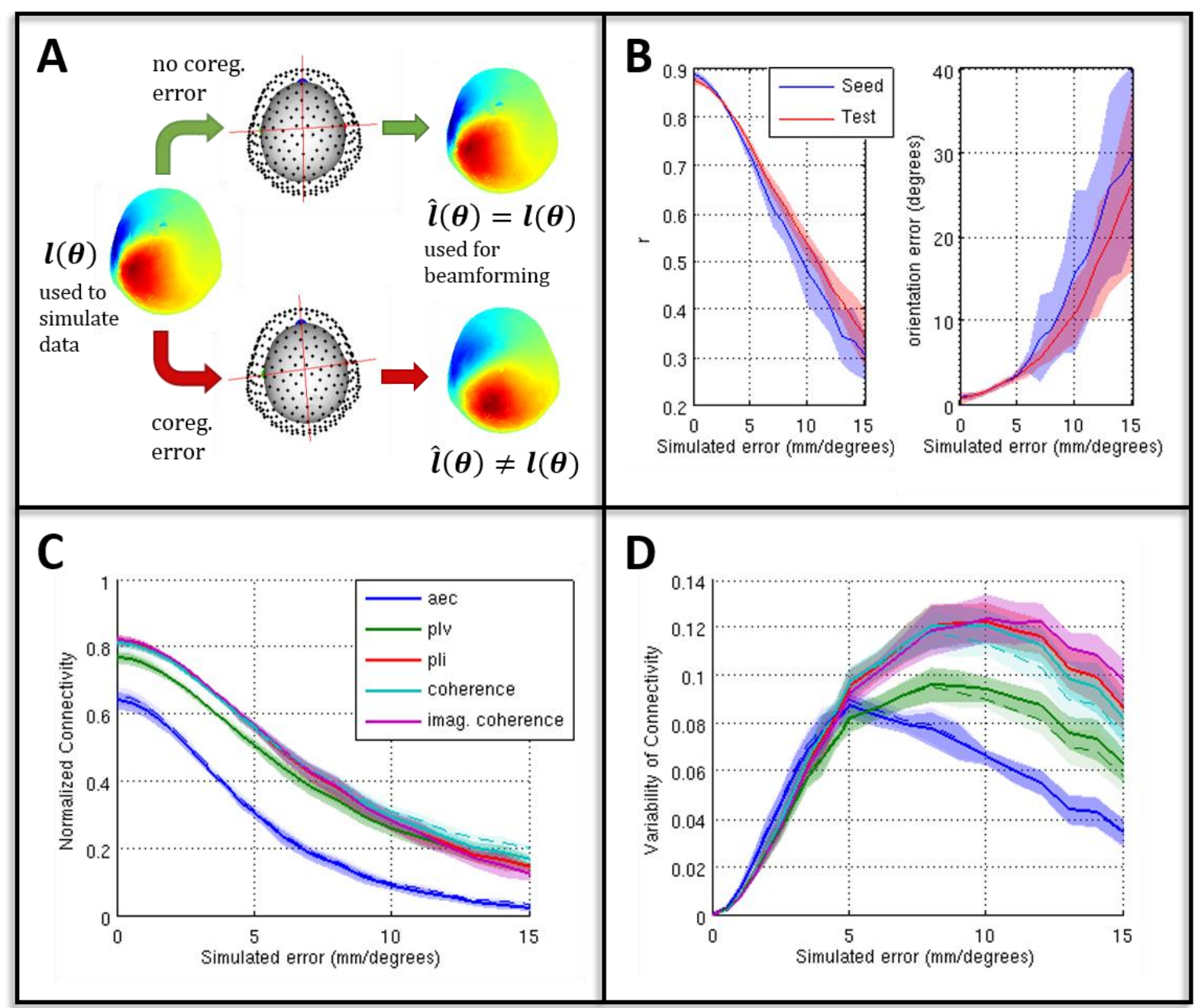

Figure 1: The effect of coregistration error. A) Schematic showing the basic principle of the coregistration error simulation. Note that coregistration error manifests as a difference between the true lead field and that used for reconstruction. B) Beamformer reconstruction accuracy (measured as correlation between $q(t)$ and $\hat{q}(t)$, left hand panel) and error in dipole orientation (right hand panel) plotted against coregistration error. C) Functional connectivity between the simulated seed and test sources versus coregistration error. D) The variability of functional connectivity across different realisations of the coregistration error. Note that in $B, C$ and $D$ the shaded regions show standard deviation across different simulated MEG datasets. In $C$ and $D$ the dashed lines refer to connectivity calculated without correcting for signal leakage. 
The results of our coregistration error simulation are shown in Figure 1. Figure $1 \mathrm{~B}$ shows the beamformer reconstruction accuracy plotted as a function of coregistration error. The left hand panel shows correlation between the simulated and reconstructed seed and test timecourses (i.e. temporal correlation between $q_{\text {seed }}(t)$ and $\hat{q}_{\text {seed }}(t)$ is given in blue and equivalently correlation between $q_{\text {test }}(t)$ and $\hat{q}_{\text {test }}(t)$ is given in red). The right hand plot shows the angular error (in degrees) between the simulated and reconstructed dipole orientation for the seed (blue) and test (red) sources. Recall that simulated coregistration error ( $x$-axes) represents both a translation and rotation (i.e. a value of 10 means $10 \mathrm{~mm}$ translation error accompanied by a $10^{\circ}$ rotational error). The error bars show standard deviation across 10 independent runs of the simulation (different realisations of simulated MEG data). As would be expected, a drop in reconstruction accuracy is observed with increasing coregistration error, and this is accompanied by an increase in the error on the reconstructed dipolar orientation. Interestingly large beamformer reconstruction errors are observed even for modest coregistration errors (e.g. an error of $10 \mathrm{~mm} /$ degrees reduces correlation between simulated and reconstructed timecourses to $\sim 0.5$ ).

Figures $1 C$ and $D$ show the effect of coregistration error on functional connectivity estimation for all 5 connectivity methods. In both plots, AEC, PLV, PLI, Coh and ICoh are shown in blue, green, red, cyan and magenta respectively. The shaded regions show the standard deviation across 10 realisations of simulated MEG data. In Figure 1C, connectivity is shown, normalised to its true value (i.e. a value of 1 would indicate perfect reconstruction of the connection strength). Figure 1D shows variability in functional connectivity; this is computed as the standard deviation of the functional connectivity estimate between the seed and test sources, made using a single MEG dataset, with 100 different coregistration errors. In other words, this variability simulates a case where the same data could be acquired but with different coregistration errors. Note again the significant effect of coregistration error; even for low ( $5 \mathrm{~mm} /$ degree) errors, the magnitude of the connectivity estimate can fall sharply (e.g. to $\sim 50 \%$ of the value with no coregistration error) and variability can be as large as $20 \%$ of the connectivity value for the phase based metrics and up to $40 \%$ of AEC. Note that variability falls with very large coregistration error since there is no genuine connectivity left.

Figure 2 shows the effect of recording duration on beamformer reconstruction accuracy and functional connectivity estimation. Figure $2 \mathrm{~A}$ shows beamformer reconstruction accuracy (upper panel) and reconstructed orientation error (bottom panel) both as a function of recording duration. The shaded area shows standard deviation across simulation iterations, the blue curves show the seed source and the red curves show the test source. Note that, in good agreement with previous work (Brookes et al., 2008), the 
beamformer reconstruction accuracy falls sharply when recording duration is less than approximately $60 \mathrm{~s}$ (see Figure 2A lower left panel). This is result of a poorly estimated covariance matrix used for beamformer estimation.

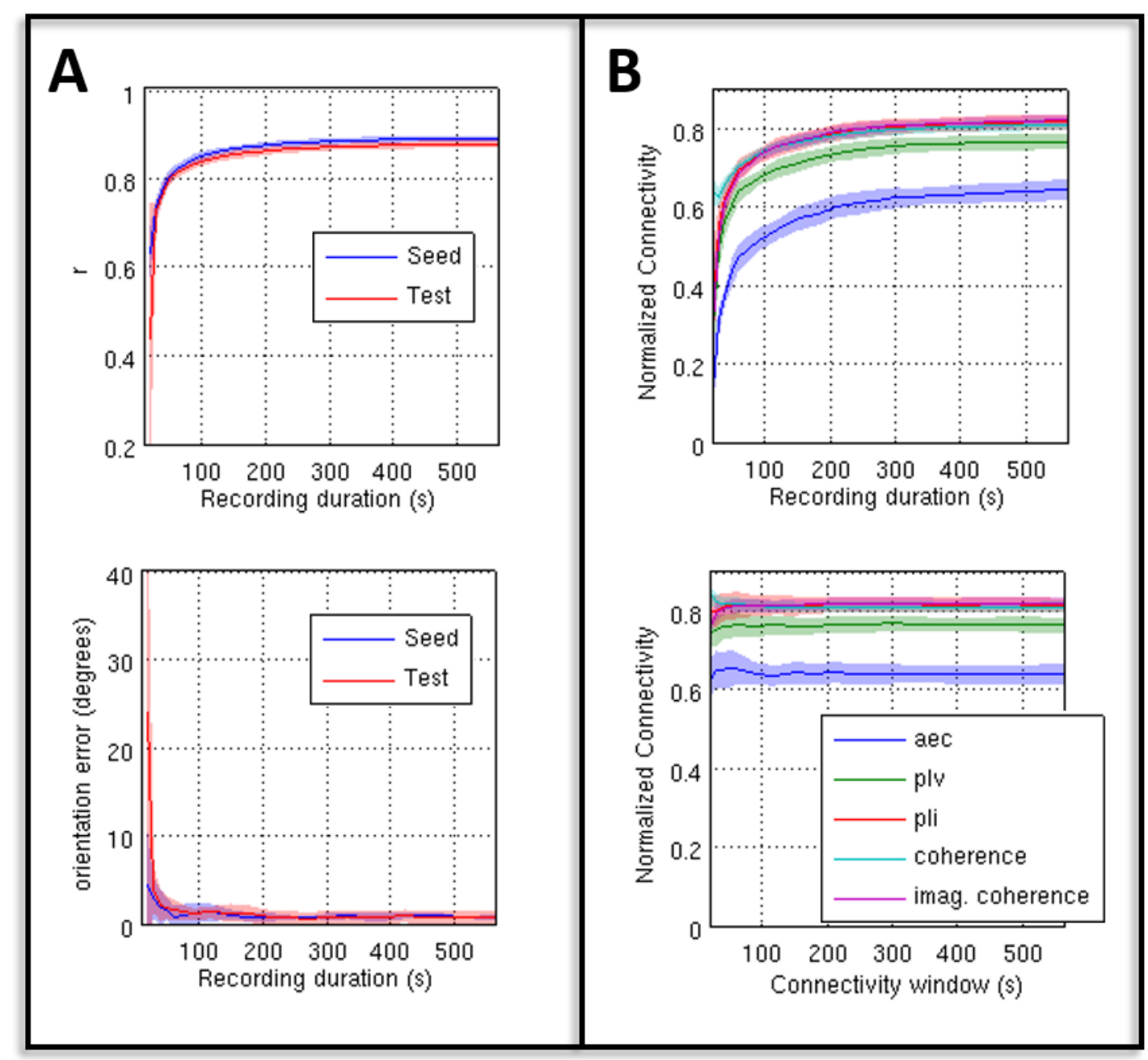

Figure 2: The effect of recording duration. A) Beamformer reconstruction accuracy (upper panel) and error in reconstructed dipole orientation (lower panel) as a function of recording duration. B) Measured connectivity between the simulated seed and test timecourses (normalised to the true value). The upper plot shows the case where beamforming and connectivity are calculated in a limited window (i.e. $x$-axis represents total recording duration). The lower plot shows a case where the beamformer parameters are calculated using $560 \mathrm{~s}$ of data but the connectivity is calculated in a limited window (i.e. $x$-axis represents only the connectivity window size).

Figure 2B (upper panel) shows connectivity estimation versus recording duration. In agreement with Figure $2 \mathrm{~A}$, connectivity estimation improves with recording duration as would be expected. However estimates tend to plateau after approximately 2 minutes of data are recorded. Note that this effect is purely a result of errors in connectivity estimation, however in real data, genuine differences in brain state (connectivity dynamics (Hutchison et al., 2013)) can also cause variability in the connectivity estimate. This will 
be addressed further below. It is noteworthy that reducing recording duration will effect connectivity estimation in two ways. First, beamformer reconstruction accuracy will decline (as shown in Figure $2 \mathrm{~A}$ ). Second, following reconstruction of timecourses $\hat{q}_{\text {seed }}(t)$ and $\hat{q}_{\text {test }}(t)$, estimation of connectivity between them will be made using fewer data points, reducing its reliability. Here, to separate these effects, we undertook measurements in which beamforming was applied using $560 \mathrm{~s}$ of data, and connectivity estimated in a smaller window. The results are shown in Figure 2B lower panel. It is clear that the effects observed in Figure 2A are dominated by the beamformer. I.e. If beamformer weights are calculated over large windows, the effect of limited data duration on connectivity (down to $20 \mathrm{~s}$ ) are practically negligible.

\section{EXPERIMENTS}

\section{Experimental methods}

Three subjects (all aged 26, 2 male) took part in the study, which was approved by the University of Nottingham Medical School Research Ethics Committee. All subjects underwent a structural MRI scan, followed by 10 resting state MEG recordings.

\section{Structural image and head-cast construction.}

An anatomical MR image of the subjects' head was acquired using a 3DTFE sequence running on a Philips Ingenia 3T system at a resolution of $1 \mathrm{~mm}^{3}$. Sequence parameters (TR $=4.5 \mathrm{~ms} ; \mathrm{TE}=1.97 \mathrm{~ms} ; \mathrm{FOV}(\mathrm{ap}, \mathrm{fh}, \mathrm{rl})=256 \times 256 \times 183 ;$ Orientation: sagittal; $\mathrm{BW}=775$ $\mathrm{Hz}$ ) were optimised such that the scalp and face surfaces were reconstructed accurately (i.e. a high bandwidth/voxel was used in order to minimise spatial distortion due to susceptibility effects around the air tissue interfaces). A single subject example is shown in Figure 3A. Following acquisition, we used this image to generate a foam head-cast, the internal surface of which was fitted to the subjects' scalp, whilst the external surface was fitted to the MEG scanner helmet (Troebinger et al., 2014). The procedure is described fully in Meyer et al. (2016). Briefly, a 3D mesh representing the outer surface of the head and face was extracted from the anatomical MRI (See Figure 3B). This virtual 3D model of the subjects' head was placed inside a virtual realisation of the MEG helmet. The headmodel was augmented with spacing elements, to optimise the distance between the scalp and sensors. In addition, a 3D representation of three head localisation coils, used for localising head position in the scanner was added, with coils placed at the nasion and left and right preauricular points. This 3D representation of the head surface, spacers, and localisation coils was 3D printed and placed inside a manufacturer-provided replica of the MEG-helmet. Finally, liquid resin was poured into the negative space between the surfaces. This expands and sets, resulting flexible foam subject-specific head-casts which can also house the localising coils (See Figure 3C). 

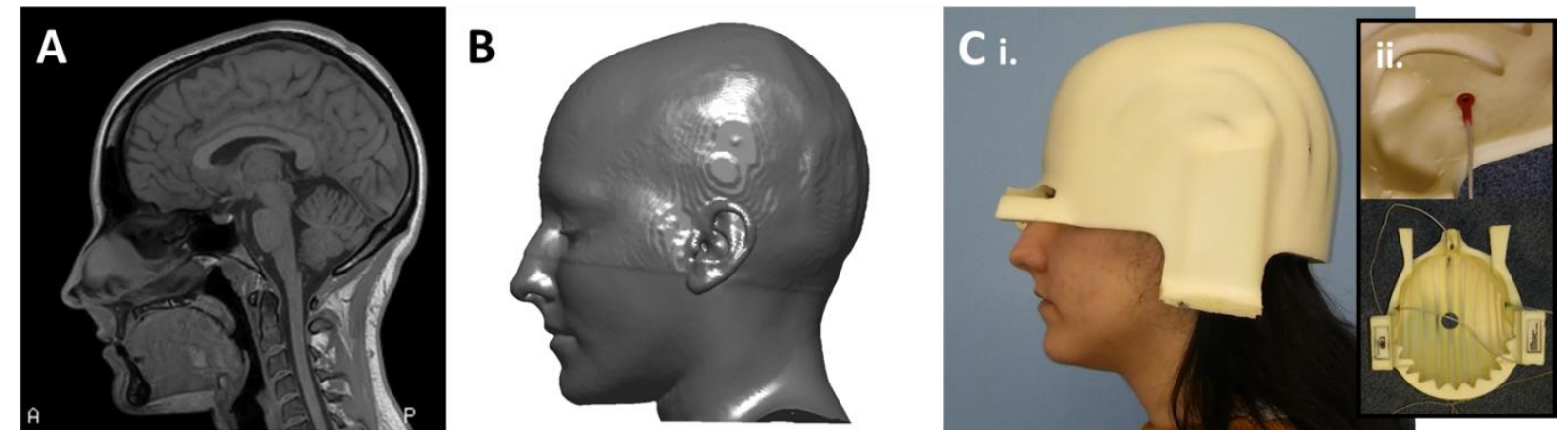

Figure 3: Subject specific foam head-cast. A) Sagittal slice showing the anatomical MRI scan. B) Scalp surface extracted from the MRI scan. C) i) Subject wearing the foam head-cast. ii) Head localisation coil in situ.

\section{MEG data acquisition}

All MEG data were acquired using a 275-channel whole head CTF system in third order synthetic gradiometer configuration. Three subjects were scanned in five separate sessions, on consecutive days. Sessions were conducted at the same time each day for the same subject. Each session comprised two recordings, one in which the subject wore the head-cast and one "standard" recording (with no head-cast). For both scans subjects were asked to sit in the MEG system, keep as still as possible and "think of nothing" while focussing on a centrally presented fixation cross (projected onto a back projection screen $\sim 40 \mathrm{~cm}$ in front of the subject). All acquisitions were 10 minutes in duration. The position of the subjects head was measured continuously by energizing 3 head localisation coils placed at the fiducial marker positions (nasion, preauricular left and right). A maximum movement of $\sim 5 \mathrm{~mm}$ was accepted. Coregistration between the MEG sensor geometry was achieved in two ways:

1) For the head-cast acquisition, the coils were held rigidly in the head-cast and their location relative to the brain anatomy was known from the virtual 3D model of the subjects head used in the head-cast design. Coregistration was therefore enabled by translating these known coordinates to the anatomical MRI.

2) For the standard acquisition, the coils were taped firmly to the subject's skin at the three fiducial locations. Prior to MEG acquisition, the coil locations were measured relative to the scalp surface using a 3D digitiser (Polhemus; Colchester, VT). Coregistration of MEG data to anatomical MRI was achieved by matching the digitised head surface to the equivalent surface extracted from the MRI.

It is noteworthy that the standard acquisition represents a procedure for coregistration used routinely in most MEG laboratories, but is nevertheless prone to inaccuracy. The head-cast has been shown as a useful methodology to minimise coregistration error (Meyer et al., 2016; Troebinger et al., 2014). 
All MEG data were inspected visually. Any segments containing artifacts such as SQUID resets or excessive muscle activity were removed (eye-blinks were not removed). Data were then frequency filtered into the $\beta$ range $(13-30 \mathrm{~Hz})$ using a least-square linear phase finite impulse response filter, implemented in Matlab. All results presented in this paper are based upon this band. The reason for this choice was a weight of literature that points towards connections in many long range networks being mediated by neural oscillations in this range (Hall et al., 2014; Hipp et al., 2012; Hunt et al., 2016).

\section{Effect of coregistration method and data duration on beamforming}

An inherent limitation when using real MEG data is that, since no underlying ground truth is known about the location or strength of an electrical source, it is challenging to track the inaccuracies inherent to reconstruction. However, the MEG signal is thought to be generated by synchronised electrical activity in pyramidal neurons, which are oriented approximately perpendicular to the cortical surface. This means that across two independent MEG sessions, whilst the strength of the activity of such neurons may change, their orientation, being defined only by anatomy, will not. For this reason, at any one brain location, reconstructed source orientation for two independent MEG sessions should be equivalent. (A similar argument has been employed in work tracking the efficacy of source reconstruction algorithms (Stevenson et al., 2014)). Using this idea, we aimed to test the effect of coregistration method (head-cast and standard) and recording duration, on beamformer reconstruction reliability across sessions.

A scalar beamformer (equivalent to that described above in our simulations section) was used to reconstruct sources at the vertices of a regular $8 \mathrm{~mm}$ grid spanning the whole of source space. For beamformer reconstruction, covariance matrices were generated independently for each acquisition. No regularisation was employed in order to maximise spatial specificity. Modelled source dipoles were limited to the tangential plane and source orientation was measured as the direction with highest signal to noise ratio. For every voxel, and each recording, this optimum orientation was stored and beamformer repeatability was inferred as the difference in orientation (measured as an angle) between pairs of sessions within each subject. (I.e. for a single voxel we compared orientation on - day 1 to day 2; - day 1 to day 3 and so on. 5 separate sessions gave 10 comparisons.) This analysis was undertaken separately for each individual, and results were averaged across people. The analysis was undertaken independently for three recording durations $(560 \mathrm{~s}, 280 \mathrm{~s}, 120 \mathrm{~s})$, and for the head-cast and standard coregistration procedures. 
Effect of coregistration method and data duration on functional connectivity

Functional connectivity was assessed between 78 cortical regions defined according to the AAL parcellation (Tzourio-Mazoyer et al., 2002). Beamformer reconstructed signals were computed at the centre of mass of each of these regions. Reconstruction was based upon either the first 560 s, 280 s or 120 s of MEG data from each recording. The beamformer was applied as described above. The 78 regional timecourses were orthogonalised using the multivariate method described in our simulations section (Colclough et al., 2015) in order to reduce the effects of signal leakage. Functional connectivity between all possible region pairs was then estimated using AEC, Coh, ICoh, PLV and PLI. For each subject and each recording, this gave a single $78 \times 78$ weighted adjacency matrix (henceforth termed the connectivity matrix) for each metric, showing beta band coupling between all regions.

We aimed to test the between session reproducibility of intra-subject measures of functional connectivity. To this end, all of the derived connectivity matrices were first reshaped into one dimensional vectors, which we term connectivity vectors. (Note that we removed the redundancy inherent in all connectivity matrices due to their symmetry, as well as the leading diagonal which represents only connectivity of a region with itself.) Following this, we measured Pearson correlation between connectivity vectors derived using MEG data acquired in different sessions in the same subject. This was repeated for all session pairs. Data acquired with and without the head-cast were treated separately and their comparison was used to assess the effect of coregistration method. Correlation values were also derived independently using either 560 s, $280 \mathrm{~s}$ or $120 \mathrm{~s}$ of MEG data to assess the effect of recording duration.

Finally, any observable effect of recording duration could result from two possible sources; 1) beamformer reconstruction error (i.e. similar to the underlying cause of the effect shown in Figure 2) or 2) genuine differences in brain state. In order to assess this, connectivity matrices were computed using two $280 \mathrm{~s}$ windows, or two $120 \mathrm{~s}$ windows, derived from the same session (e.g. within a single $560 \mathrm{~s}$ recording in one session, we compare a connectivity matrix acquired in the first $280 \mathrm{~s}$, to a connectivity matrix acquired in the final $280 \mathrm{~s}$ ). To eliminate the effect of the beamformer reconstruction, the same beamformer weighting parameters were used (i.e. beamformer weights were derived using data acquired across the whole recording (encapsulating both time windows)). It was reasoned that if an effect of data duration was observed in the between session comparisons, but not the within session comparisons, then such an effect would likely be due to the beamformer reconstruction. However if an effect was measurable in both the between session and within session comparisons, this likely represents differences in brain state. 


\section{Statistical testing}

In order to assess whether the effects of head-cast or data recording duration were significant, a permutation test was employed. First, the values of correlation between connectivity vectors for two conditions (e.g. head-cast or standard), for all possible session pairs within a subject, and for all subjects, were averaged and the difference calculated. This difference was then compared to an empirical null distribution. To generate the null, we reasoned that if no effect was measurable then the condition labels apportioned to different recordings would have no meaning. These were therefore switched randomly and the difference between 'sham' conditions calculated. Comparison of the true value with the empirical null then gave rise to a $p$-value. Significance was assigned for $p$-values less than 0.05 .

\section{Experimental Results}

Head movement was acceptable for all subjects and all sessions; without the head-cast, subjects moved $2.5 \pm 1.7 \mathrm{~mm}$ (mean \pm standard deviation over sessions and subjects). With the head-cast, subjects moved $0.4 \pm 0.2 \mathrm{~mm}$.

Figure 4 shows the effect of recording duration and coregistration method on the repeatability of the beamformer reconstructed orientation. The upper plot shows a histogram of orientation differences between MEG sessions. I.e. the $x$-axis represents the angular difference in orientation of a source at a single voxel in a single subject scanned in two separate sessions. The $y$-axis shows the number of voxels. The centre plot collapses data over all pairs of recording sessions and subjects. The smaller plots (inset) show the case for each subject individually. The red line shows the case for head-cast coregistration; the black line shows the case for standard coregistration. As expected, in both cases, beamformer projected source orientations are consistent between sessions (histogram centred about zero). However note that the spread of angles is less when coregistration is achieved using a head-cast. This observation is further depicted by the bar charts in Figure 3B, which show the percentage of voxels with an angular discrepancy between sessions of less than 10 degrees. The three plots show the case for $120 \mathrm{~s}$ recordings, $280 \mathrm{~s}$ recordings and $560 \mathrm{~s}$ recordings. Note the significant increase in voxel count when using the headcast (significance tested using a permutation test). These data confirm previous reports (Meyer et al., 2016; Troebinger et al., 2014) in demonstrating the utility of head-cast technology to improve the within subject consistence of MEG reconstructions. Also noteworthy is the effect of recording duration, with a lower angle error with longer recordings; this is particularly noticeable for the data recorded with a head-cast. 

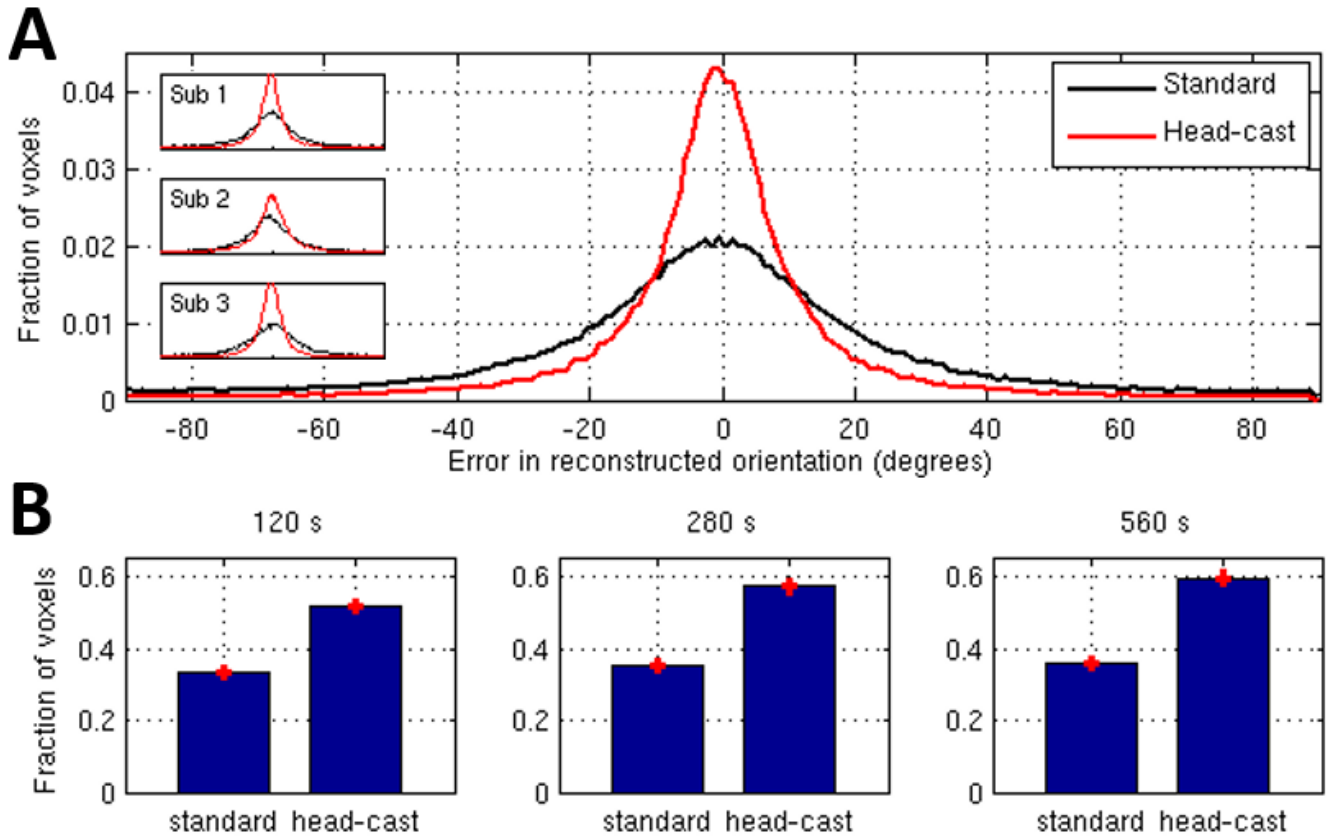

Figure 4: Between session consistencies of beamformer reconstructed source orientation.

A) Histogram showing orientation difference (measured as an angle in degrees) in beamformer source reconstruction between sessions. The red curve shows the case with head-cast coregistration and the black curve shows the case with standard coregistration. The main plot shows all subjects combined; inset plots shows the three subjects separately on the same scale. B) Bar charts showing the percentage of voxels (collapsed across subjects) with an angle error of less than 10 degrees. The three plots show the case for three different recording durations, $120 \mathrm{~s}, 280 \mathrm{~s}$ and $560 \mathrm{~s}$.

Figure 5A shows a visual comparison of the connectivity matrices for all three subjects and each of the five connectivity metrics. Note that these matrices have been averaged across all sessions (head-cast and standard) within each subject using all $560 \mathrm{~s}$ of data. The 3D brains show a graphical representation of the top $10 \%$ of connections. Figure 5B shows the between session correlation of measured connectivity vectors for different recording durations. The 5 plots show the 5 separate connectivity metrics (AEC, Coh, ICoh, PLI and PLV). The red lines show the case with head-cast coregistration and the blue lines show the case with standard coregistration. A star $(*)$ indicates a significant effect of coregistration method. Note that between session correlation is higher with the head-cast for all metrics, but this is only significant when using the phase based measures Coh, ICoh and PLV. There is also a notable difference between metrics with AEC being the most reliable measure across sessions, and PLI the least reliable. This is in agreement with other published work (Colclough et al., 2016). By far the biggest effect comes from data recording duration, with reproducibility across sessions increasing markedly when moving from $120 \mathrm{~s}$ to $560 \mathrm{~s}$ recordings. 
A

Subject 1
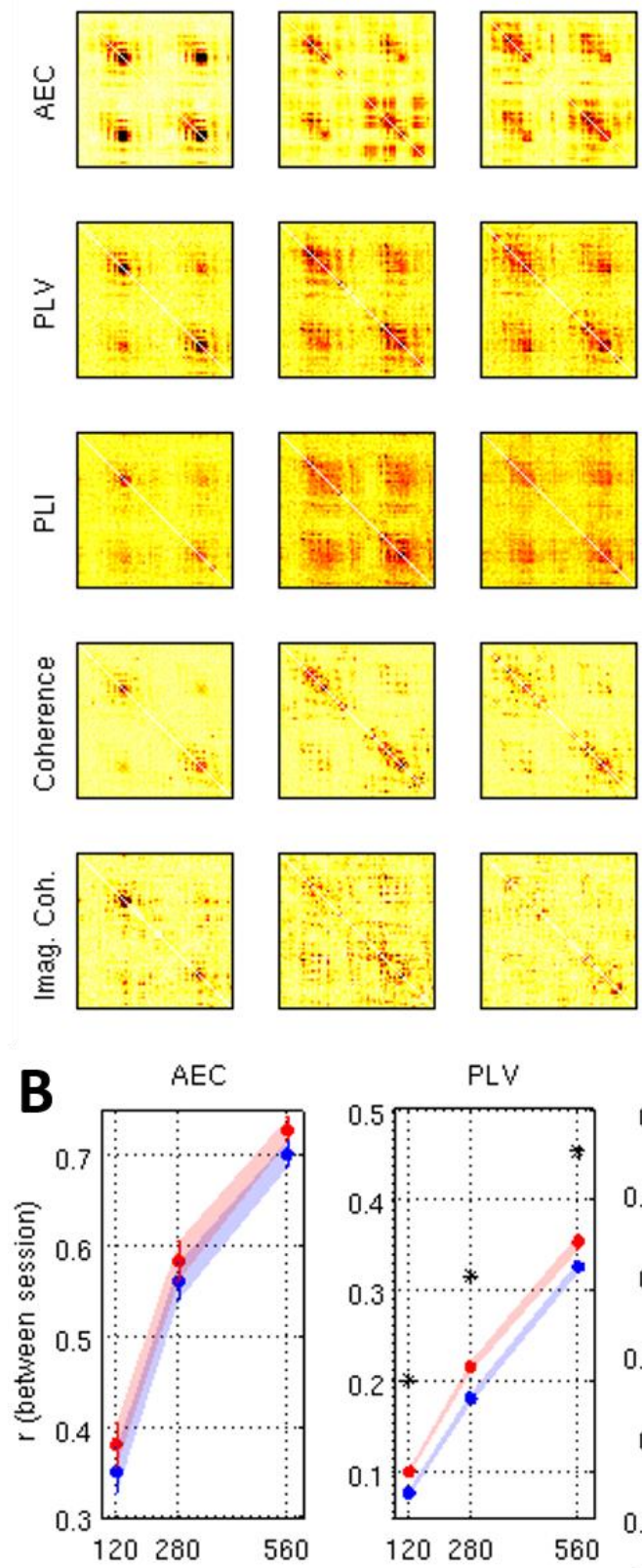

- Standard

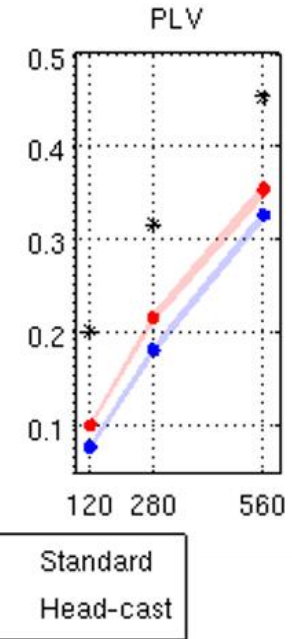

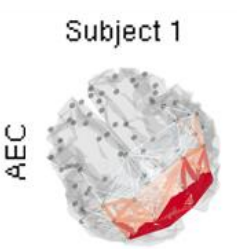
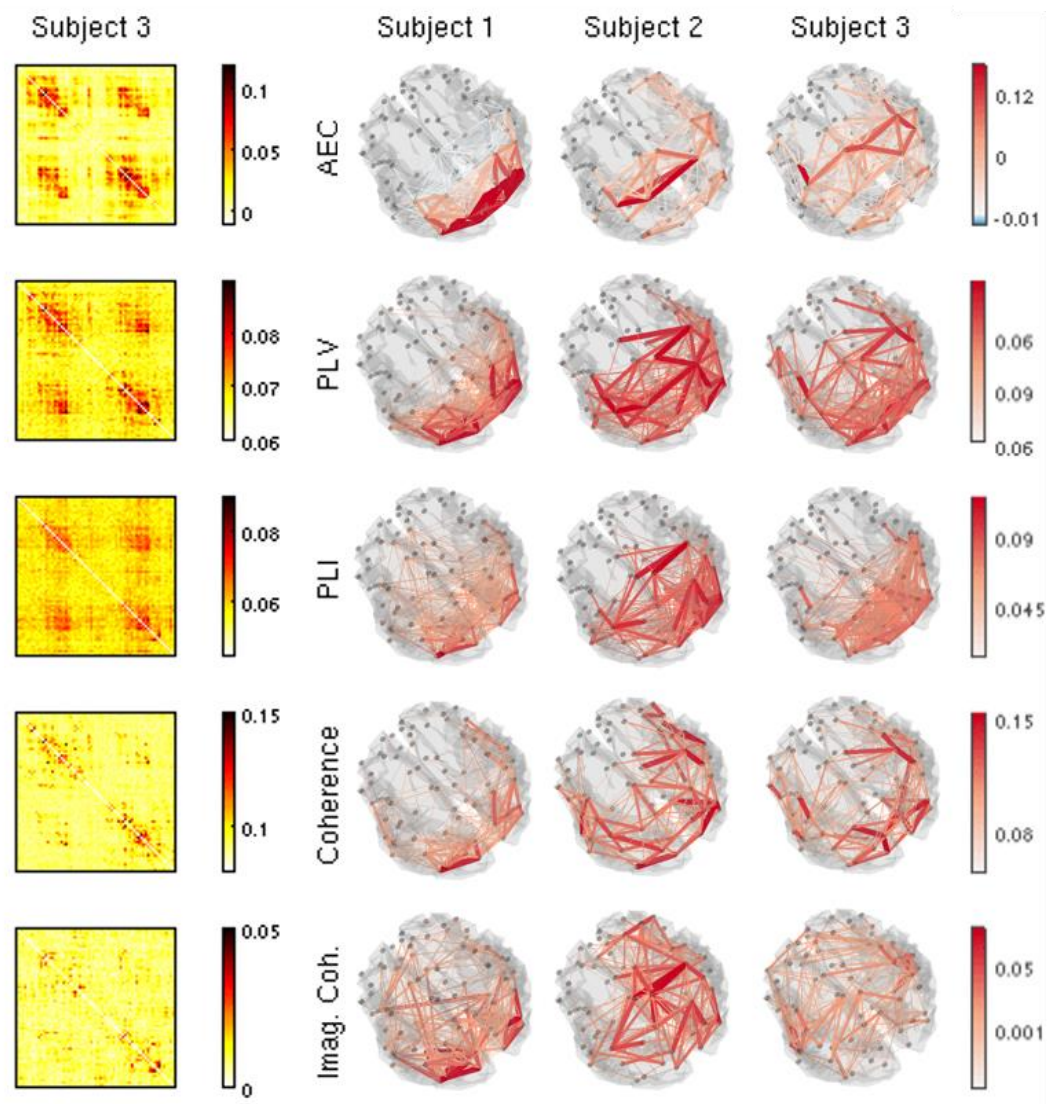

PLI
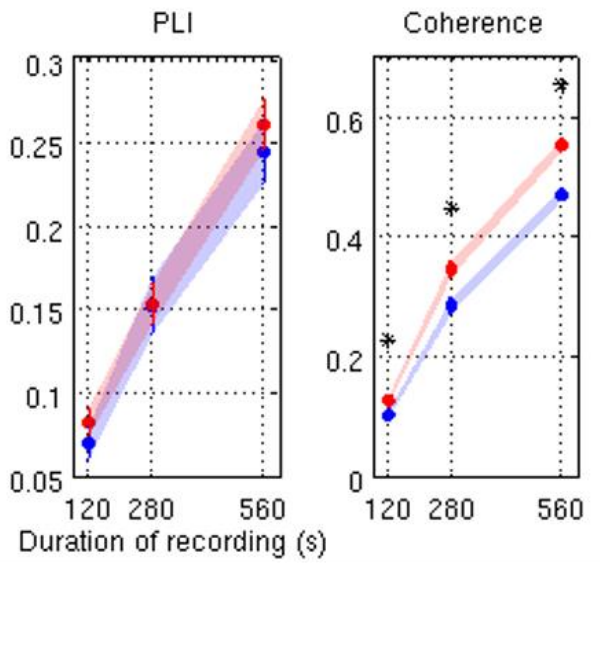

Imaginary Coherence

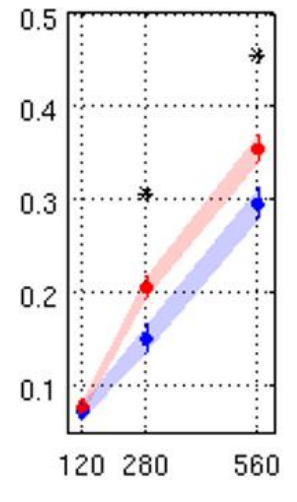

Figure 5: Between session consistency of functional connectivity measurement. A) Connectivity matrices for all three subjects and each of the five connectivity metrics. 3D brain images show the top 10 percent of connections. B) Between session correlation of measured connectivity vectors as a function of duration of a recording. The 5 plots show the 5 separate metrics (AEC, Coh, ICoh, PLI and PLV). The red lines show the case with the head-cast coregistration and the blue lines show the case with standard coregistration. A star $(*)$ indicates a significant effect of coregistration. 

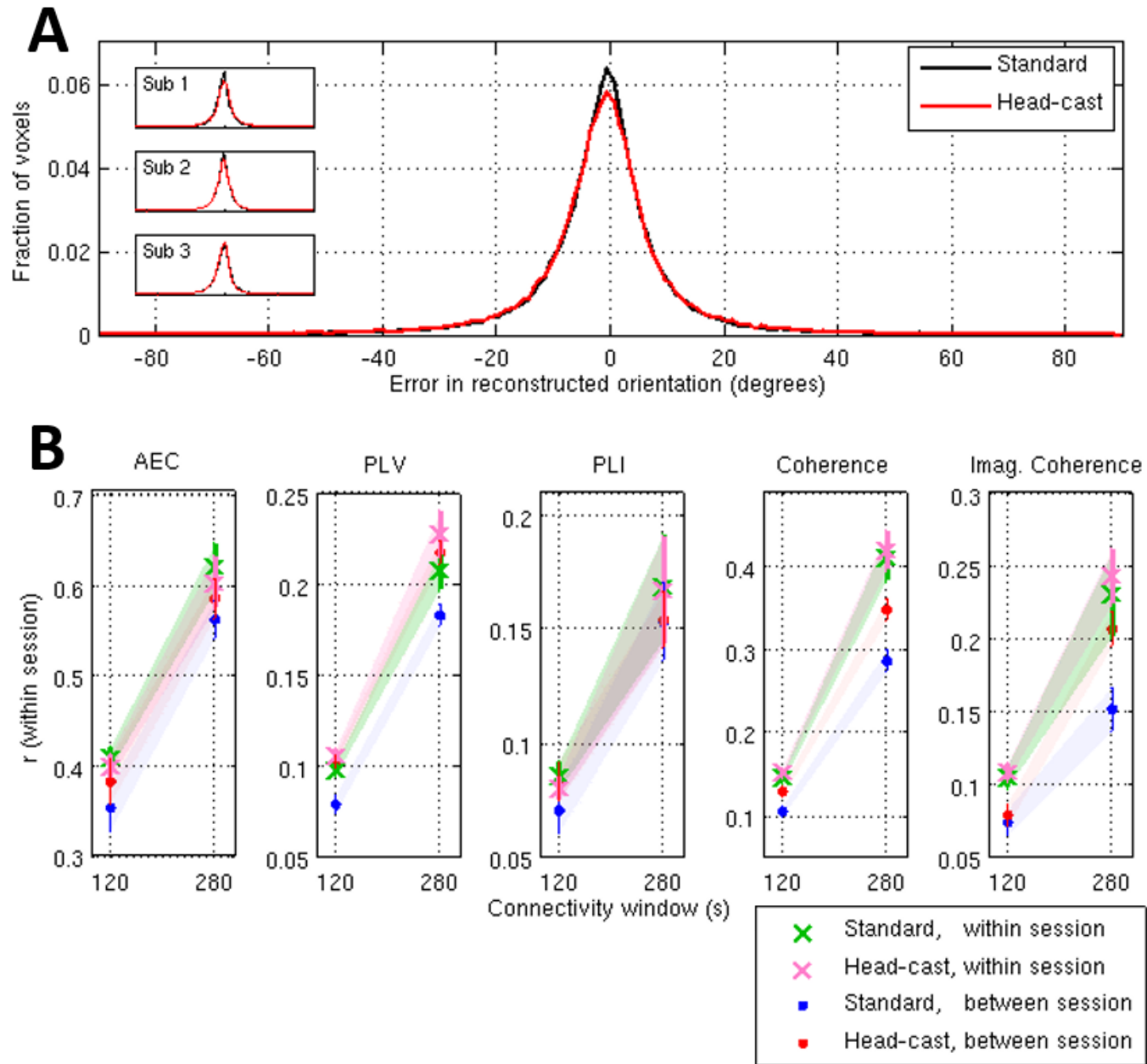

Figure 6: Within session consistency of orientation reconstruction and functional connectivity. A) Histogram showing orientation difference (measured as an angle in degrees) in beamformer source reconstruction for multiple windows within a single session. The main plot shows all subjects combined; inset plots shows the three subjects separately on the same scale. B) Correlation of connectivity vectors measured in multiple time windows in the same session. The 5 plots show the 5 separate connectivity metrics AEC, Coh, ICoh, PLI and PLV). The pink crosses show the case with the head-cast coregistration and the green crosses show the case with standard coregistration. For comparison, the red and blue dots show the between session results (copied from Figure 5B).

Figure 6 shows the within session consistency of both beamformer orientation reconstruction and connectivity calculation. Figure $6 \mathrm{~A}$ shows a histogram of orientation differences between different time windows within the same MEG session. Here, beamformer reconstructed dipole orientation has been reconstructed within the first $280 \mathrm{~s}$, and the final 280s of each MEG recording and compared (i.e. Figure 6A shows orientation consistency within a MEG session, as distinct from between MEG sessions as was shown in Figure $3 \mathrm{~A}$ ). The red line shows the case with head-cast coregistration whereas the black line shows the standard coregistration. Interestingly, the effect of the head-cast that was 
apparent in the between session recording has been eliminated. This shows that, whilst between session consistency is improved by using the head-cast, within session consistency is approximately equal - this would be expected since the head-cast is predominantly improving coregistration error which does not change within a session.

Figure 6B (green and pink crosses) shows the correlation between connectivity vectors measured using different time windows within the same recording session. Note here that beamformer parameters are the same for all windows and hence the effect of the beamformer is removed. The pink lines show within session connectivity correlation with a head-cast and the green lines show within session connectivity correlation without a head-cast. For comparison, the red and blue lines show the equivalent between session values for comparison (i.e. these are the same as in Figure 5). Note that the effect of head-cast coregistration on the within session correlation is mostly eliminated. An improvement in reproducibility for within session, compared to between session is observed, but this is most apparent when comparing between session correlation using standard coregistration, with within session correlation. Most importantly, the effect of recording duration remains. This implies that the significant effect of recording duration on the reliability of functional connectivity (shown in Figure 5) is not down to beamformer reconstruction accuracy, but rather represents changes in brain state, either between session, or between time windows in the same session.

Finally, Figure 7 shows between session correlations of connectivity, independently for each AAL region. Here, rather than measure correlation between sessions over an entire connectivity matrix, correlation is measured column by column (i.e. for each AAL region independently). Red shows brain regions with high between session reproducibility and blue shows regions with low between session reproducibility. The left hand column shows the case for standard coregistration and the right hand column shows the case for headcast coregistration. The three rows show three different recording durations. Panel A shows the case for AEC and B shows the case for Coherence (selected since it proved to be the most reliable (across sessions) of the phase based metrics). In agreement with results shown in Figure 5, the significant effect of head-cast can be seen for the coherence measurements. In addition, the comparatively large effect of recording duration can be seen clearly. It is noteworthy that the highest between session reliability is observed in the parietal and occipital lobes and this is consistent with functional connectivity in beta band being dominated by these brain areas, as shown in Figure 5 (see also Hunt et al., 2016). However it is important to point out that this is likely because results are derived from resting state data. It is well known that cognitive tasks induce strong transient activity and connectivity in frontal areas (Fox et al., 2005) and our own previous studies 
support this (Brookes et al., 2014). However, the signal to noise ratio of these frontal networks is typically lower. We would therefore not only speculate that the advantages of data recording duration and head-cast shown here, dominated by the posterior brain areas, would be observed in frontal regions for task positive data. But we also suggest that these advantages are potentially more important in task positive studies in order to compensate for their lower SNR.

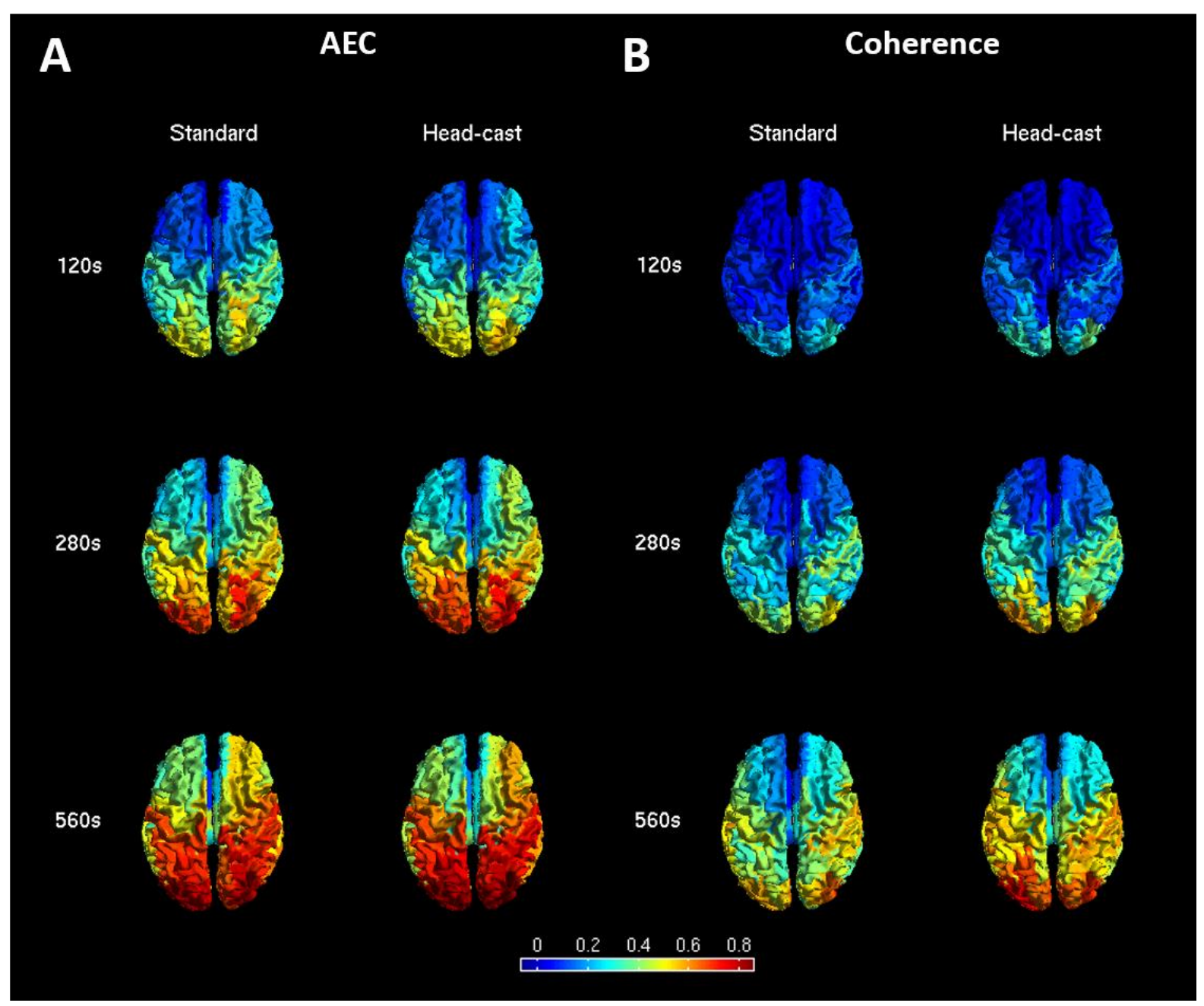

Figure 7: Between session correlation of functional connectivity, on a 'per region' basis.

A) Between session correlations of connectivity profiles measured using AEC in the beta band. The left hand column shows the case for standard coregistration. The right hand column shows the case for head-cast coregistration. The three rows show three different recording durations. B) Equivalent to $A$, but for Coherence. 


\section{DISCUSSION}

We aimed to test how practical aspects of experimental design affect the intra-subject repeatability of MEG functional connectivity measurement. Specifically we assessed the effects of coregistration method and data recording duration on the between session reliability of network estimation. Our results showed that the use of a foam head-cast, which is known to improve coregistration accuracy (Meyer et al., 2016; Troebinger et al., 2014), increased significantly the between session repeatability of both MEG reconstruction accuracy and connectivity estimation. This was the case for all MEG connectivity metrics, but was strongest for phase based methods. Further, we showed that recording duration is a critical parameter, with large improvements in repeatability apparent when using ten minute, compared to five minute recordings. Further analyses suggest that the origin of this effect is not underpinned by technical aspects of source reconstruction, but rather by a genuine effect of brain state.

Coregistration error has been identified as one of the most significant problems with MEG source reconstruction. Perhaps counter-intuitively, as the signal to noise ratio of MEG measurements has increased (i.e. as system architecture has improved), the sensitivity of source reconstruction, particularly using adaptive methods such as beamforming, to coregistration accuracy has also increased (Boto et al., 2016). This means that in modern multi-channel systems, even modest errors in knowledge of sensor location and orientation relative to brain anatomy generate a significant degradation in reconstruction accuracy. The extent of this problem is shown clearly by our simulations. We show that even relatively small errors have a significant impact on both beamformer reconstruction and connectivity estimation; for example, for simulated sources in left and right motor cortex, a translational error in coregistration of $5 \mathrm{~mm}$, coupled with a rotational error of $5^{\circ}$ reduces reconstruction accuracy from $88 \%$ to $70 \%$. Such errors propagate to connectivity estimation, and have the effect of reducing the measured magnitude of functional connectivity and increasing the variability of connectivity over simulated recordings.

In real data, assessing the effect of coregistration error is challenging since the ground truth of precisely where the head is with respect to the MEG helmet is never known. Nevertheless, by exploiting the fact that source orientation, which is defined purely by brain anatomy, should be constant across multiple MEG acquisitions, we were able to show that source reconstruction accuracy is improved significantly by the use of head-cast technology. In agreement with our simulations, this improvement is translated to connectivity estimation with between session reproducibility of connectivity significantly higher when using the head-cast for 3 of our 5 connectivity metrics. The biggest improvement was for coherence which showed approximately a $20 \%$ improvement in 
between session reliability when using the head-cast compared to standard coregistration. Interestingly, whilst a significant effect of coregistration method was observed for 3 out of 4 phase based metrics, the improvement in between session reliability for AEC failed to reach statistical significance. There are two potential reasons for this: first, the between session reliability of AEC is already high ( $70 \%$, which is in line with previous estimates (Colclough et al., 2016)). The effect of the head-cast may therefore be minimised. Second, it could be that phase based metrics are more susceptible to coregistration error than amplitude based metrics. It is noteworthy that of the 4 phase metrics, PLI also failed to show a significant effect of head-cast. However, of all the measures tested here, PLI showed the lowest between session reliability ( $25 \%$ - again in line with previous results (Colclough et al., 2016)).

Our coregistration results show clearly that both beamformer reconstruction and connectivity estimation are improved by the head-cast method of coregistration. However, whether head-cast technology should become commonplace for all MEG acquisitions is debatable. It is important to note that the three individuals who took part in our study were all experienced subjects. (Indeed this was evidenced by the relatively low levels of movement when they were scanned without a head-cast.) All three tolerated the use of the head-cast well, however whether scanner naïve individuals would cope so well is unclear. The head-casts are made from foam and are therefore relatively soft and comfortable to wear. However, they do restrict head movement when the subject is in the scanner, and subjects can become warm. This means, potentially, that some people (particularly infants, or some patient groups) may find wearing a head-cast worrisome, or claustrophobic. This could limit their utility for broader use. A second limitation is cost: The head-cast construction process is relatively involved and costly, and this may be prohibitive for studies with large subject numbers; the modification of the design to a cheaper method, perhaps using only subject specific foam pads rather than a whole headcast, might be advantageous. Finally, even when using a head-cast, coregistration error can arise due to, for example, movement of the head localisation coils inside the cast, localisation of the magnetic signature of the coils, or even differences in brain position within the skull between a seated MEG measurement and a supine MRI measurement, meaning that coregistration will always involve some inherent error. Overall, our results certainly provide sufficient motivation to suggest that novel methods for coregistration should be sought for future MEG investigations, particularly if individual subject results are required. However, whether those methods should be limited to head-casts, or other (e.g. optical) coregistration techniques remains a topic for future work. 
The effect of recording duration manifests in two ways. 1) There is a technical limitation generated when reconstructing data and measuring functional connectivity using a reduced number of data points. This manifests firstly as an error in the data covariance matrix used for beamformer weights generation (Brookes et al., 2008); fewer data points mean a larger covariance matrix error, and ultimately a lower beamformer projected power. Second, when estimating functional connectivity itself, most correlative or coherence based measures are highly sensitive to the number of degrees of freedom in the timecourses used to generate them. 2) In addition to the technical limitation, the well characterised dynamic nature of functional connectivity, which changes over seconds, and even milliseconds (Baker et al., 2014; Brookes et al., 2014; Chang and Glover, 2011; Hutchison et al., 2013; G C O'Neill et al., 2015 b; O'Neill et al., 2016) must be considered, since there is a question regarding how well a short time window can capture the canonical MEG networks, if those networks are constantly changing across multiple time-scales. Here, we first probed this question in simulation; our simulations do not take into account brain state and for this reason only the technical limitations are addressed. Our results show that beamformer reconstruction and connectivity are indeed impacted by recording duration; but mainly for recording durations below $100 \mathrm{~s}$ (see Figure 2). Above $100 \mathrm{~s}$, these effects tend to plateau, and so for the typical windows that might be used for resting state MEG acquisition (several minutes), one might infer that recording duration would have little effect. Our simulations also showed that these effects are dominated by inaccuracies in beamforming, rather than the reduced degrees of freedom available for functional connectivity measurement. [As an aside, it should be noted that these simulations are based on the beta band only; the technical limitations on data recording duration are related not only to the time window, but also to the bandwidth of the data, since the number of temporal degrees of freedom in a timecourse is given by twice the product of the bandwidth and the window duration. This means that for frequency bands with smaller bandwidth, the curves in Figure 2 would tend to their plateau value slower.]

In apparent contradiction to the evidence from the simulations, in real data we showed that changing recording duration (above $100 \mathrm{~s}$ ) had by far the largest effect on between session repeatability of connectivity. This therefore implies that the underlying source of this effect is related not to technical limitations, but rather to genuine problems with characterising brain state. In support of this, we also measured within session correlation between connectivity matrices, where data within two time windows used for connectivity estimation were reconstructed using the same beamformer weighting parameters; this, in effect, removes the technical limitations associated with beamformer reconstruction using limited data. Our results show that within session (beamformer limitations removed) and between session (beamformer limitations present) correlation between connectivity 
matrices was approximately equivalent. This, coupled with the evidence from simulation that using a $17 \mathrm{~Hz}$ (beta) bandwidth, $100 \mathrm{~s}$ should be sufficient to characterise adequately connectivity from a technical point of view, suggests that the significant variation of connectivity reliability with recording duration is a genuine effect; put simply, short recordings are simply inefficient at capturing the canonical MEG network in a single subject. It follows directly that, to maximise intra- (and indeed inter-) subject reliability in future resting state MEG studies, data recording durations should be a minimum of 10 minutes, and even longer (e.g. $15 \mathrm{~min}$ ) recordings may provide further advantages.

\section{CONCLUSION}

The study of functional connectivity using MEG is an expanding area, the importance of which is evidenced by recent demonstrations of utility in clinical studies. However, previous reports suggest that whilst comparisons of MEG derived connectivity between large group studies are robust, single session recordings, even in the same individual, lack repeatability. In this paper, we aimed to test how two practical aspects of experimental design (coregistration method and recording duration) affect intra-subject repeatability. Our results showed that the use of a foam head-cast increased the between session repeatability of both MEG reconstruction accuracy and connectivity estimation. Further, we showed that recording duration is a critical parameter, with large improvements in repeatability apparent when using ten minute, compared to five minute recordings. Our results provide important insights on experimental design and will be useful for future MEG connectivity studies.

\section{ACKNOWLEDGEMENTS}

This work was funded by a Medical Research Council (MRC) New Investigator Research Grant (MR/M006301/1) and an associated studentship (to LL) funded by the University of Nottingham. We also acknowledge Medical Research Council Partnership Grant (MR/K005464/1).

\section{REFERENCES}

Baker, A.P., Brookes, M.J., Rezek, I.A., Smith, S.M., Behrens, T., Probert Smith, P.J., Woolrich, M., 2014. Fast transient networks in spontaneous human brain activity. Elife 3, e01867. doi:10.7554/eLife.01867

Beckmann, C.F., DeLuca, M., Devlin, J.T., Smith, S.M., 2005. Investigations into restingstate connectivity using independent component analysis. Philos Trans R Soc L. B Biol Sci 360, 1001-1013. doi:10.1098/rstb.2005.1634

Boto, E., Bowtell, R., Krüger, P., Fromhold, T.M., Morris, P.G., Meyer, S.S., Barnes, G.R., Brookes, M.J., 2016. On the Potential of a New Generation of Magnetometers for MEG: A Beamformer Simulation Study. PLoS One 11, e0157655. doi:10.1371/journal.pone.0157655

Brookes, M.J., O'Neill, G.C., Hall, E.L., Woolrich, M.W., Baker, A., Palazzo Corner, S., Robson, S.E., Morris, P.G., Barnes, G.R., 2014. Measuring temporal, spectral and 
spatial changes in electrophysiological brain network connectivity. Neuroimage 91 , 282-299. doi:10.1016/j.neuroimage.2013.12.066

Brookes, M.J., Tewarie, P.K., Hunt, B.A.E., Robson, S.E., Gascoyne, L.E., Liddle, E.B., Liddle, P.F., Morris, P.G., 2016. A multi-layer network approach to MEG connectivity analysis. Neuroimage 132, 425-438. doi:http://dx.doi.org/10.1016/j.neuroimage.2016.02.045

Brookes, M.J., Vrba, J., Robinson, S.E., Stevenson, C.M., Peters, A.M., Barnes, G.R., Hillebrand, A., Morris, P.G., 2008. Optimising experimental design for MEG beamformer imaging. Neuroimage 39, 1788-1802. doi:10.1016/j.neuroimage.2007.09.050

Brookes, M.J., Woolrich, M.W., Barnes, G.R., 2012. Measuring functional connectivity in MEG: a multivariate approach insensitive to linear source leakage. Neuroimage 63, 910-920. doi:10.1016/j.neuroimage.2012.03.048

Brookes, M.J., Zumer, J.M., Stevenson, C.M., Hale, J.R., Barnes, G.R., Vrba, J., Morris, P.G., 2010. Investigating spatial specificity and data averaging in MEG. Neuroimage 49, 525-538. doi:10.1016/j.neuroimage.2009.07.043

Chang, C., Glover, G.H., 2011. Variable-density spiral-in/out functional magnetic resonance imaging. Magn Reson Med 65, 1287-1296. doi:10.1002/mrm.22722

Colclough, G.L., Brookes, M.J., Smith, S.M., Woolrich, M.W., 2015. A symmetric multivariate leakage correction for MEG connectomes. Neuroimage 117, 439-448. doi:http://dx.doi.org/10.1016/j.neuroimage.2015.03.071

Colclough, G.L., Woolrich, M.W., Tewarie, P.K., Brookes, M.J., Quinn, A.J., Smith, S.M., 2016. How reliable are MEG resting-state connectivity metrics? Neuroimage 138, 284-293. doi:http://dx.doi.org/10.1016/j.neuroimage.2016.05.070

Corbetta, M., Akbudak, E., Conturo, T.E., Snyder, A.Z., Ollinger, J.M., Drury, H.A., Linenweber, M.R., Petersen, S.E., Raichle, M.E., Van Essen, D.C., Shulman, G.L., 1998. A common network of functional areas for attention and eye movements. Neuron 21, 761-773.

Finn, E.S., Shen, X., Scheinost, D., Rosenberg, M.D., Huang, J., Chun, M.M., Papademetris, X., Constable, R.T., 2015. Functional connectome fingerprinting: identifying individuals using patterns of brain connectivity. Nat Neurosci 18, 16641671.

doi:10.1038/nn.4135http://www.nature.com/neuro/journal/v18/n11/abs/nn.4135.h tml\#supplementary-information

Fox, M.D., Raichle, M.E., 2007. Spontaneous fluctuations in brain activity observed with functional magnetic resonance imaging. Nat Rev Neurosci 8, 700-711. doi:10.1038/nrn2201

Fox, M.D., Snyder, A.Z., Vincent, J.L., Corbetta, M., Van Essen, D.C., Raichle, M.E., 2005. The human brain is intrinsically organized into dynamic, anticorrelated functional networks. Proc Natl Acad Sci U S A 102, 9673-9678. doi: $10.1073 /$ pnas. 0504136102

Fraschini, M., Demuru, M., Crobe, A., Marrosu, F., Stam, C.J., Hillebrand, A., 2016. The effect of epoch length on estimated EEG functional connectivity and brain network organisation. J. Neural Eng. 13, 36015. doi:10.1088/1741-2560/13/3/036015

Friston, K.J., 1998. The disconnection hypothesis. Schizophr. Res. 30, 115-125.

Friston, K.J., 1994. Functional and effective connectivity in neuroimaging: A synthesis. Hum. Brain Mapp. 2, 56-78. doi:10.1002/hbm.460020107

Guggisberg, A.G., Honma, S.M., Findlay, A.M., Dalal, S.S., Kirsch, H.E., Berger, M.S., Nagarajan, S.S., 2008. Mapping functional connectivity in patients with brain lesions. Ann Neurol 63, 193-203. doi:10.1002/ana.21224

Hall, E.L., Robson, S.E., Morris, P.G., Brookes, M.J., 2014. The relationship between MEG and fMRI. Neuroimage 102 Pt 1, 80-91. doi:10.1016/j.neuroimage.2013.11.005

Hipp, J.F., Hawellek, D.J., Corbetta, M., Siegel, M., Engel, A.K., 2012. Large-scale cortical correlation structure of spontaneous oscillatory activity. Nat Neurosci 15, 884-890.

doi:http://www.nature.com/neuro/journal/v15/n6/abs/nn.3101.html\#supplementar $\mathrm{y}$-information 
Hunt, B.A.E., Tewarie, P.K., Mougin, O.E., Geades, N., Jones, D.K., Singh, K.D., Morris, P.G., Gowland, P.A., Brookes, M.J., 2016. Relationships between cortical myeloarchitecture and electrophysiological networks. Proc. Natl. Acad. Sci. 113, 13510-13515. doi:10.1073/pnas.1608587113

Hutchison, R.M., Womelsdorf, T., Allen, E.A., Bandettini, P.A., Calhoun, V.D., Corbetta, M., Della Penna, S., Duyn, J.H., Glover, G.H., Gonzalez-Castillo, J., Handwerker, D.A., Keilholz, S., Kiviniemi, V., Leopold, D.A., de Pasquale, F., Sporns, O., Walter, M., Chang, C., 2013. Dynamic functional connectivity: promise, issues, and interpretations. Neuroimage 80, 360-378. doi:10.1016/j.neuroima

ge.2013.05.079

Kessler, D., Angstadt, M., Welsh, R.C., Sripada, C., 2014. Modality-Spanning Deficits in Attention-Deficit/Hyperactivity Disorder in Functional Networks, Gray Matter, and White Matter. J. Neurosci. 34, 16555-16566. doi:10.1523/JNEUROSCI.315614.2014

Maldjian, J.A., Davenport, E.M., Whitlow, C.T., 2014. Graph theoretical analysis of resting-state MEG data: Identifying interhemispheric connectivity and the default mode. Neuroimage 96, 88-94. doi:10.1016/j.neuroimage.2014.03.065

Meyer, S.S., Bonaiuto, J., Lim, M., Rossiter, H., Waters, S., Bradbury, D., Bestmann, S., Brookes, M., Callaghan, M.F., Weiskopf, N., Barnes, G.R., 2016. Flexible head-casts for high spatial precision MEG. Submiss.

O'Neill, G.C., Barratt, E.L., Hunt, B.A.E., Tewarie, P.K., Brookes, M.J., 2015. Measuring electrophysiological connectivity by power envelope correlation: a technical review on MEG methods. Phys. Med. Biol. 60, R271-R295. doi:10.1088/0031$9155 / 60 / 21 / R 271$

O’Neill, G.C., Bauer, M., Woolrich, M.W., Morris, P.G., Barnes, G.R., Brookes, M.J., 2015. Dynamic recruitment of resting state sub-networks. Neuroimage 115, 85-95. doi:10.1016/j.neuroimage.2015.04.030

O'Neill, G.C., Tewarie, P.K., Colclough, G.L., Gascoyne, L.E., Hunt, B.A.E., Morris, P.G., Woolrich, M.W., Brookes, M.J., 2016. Measurement of dynamic task related functional networks using MEG. Neuroimage. doi: $10.1016 / j$.neuroimage.2016.08.061

Palaniyappan, L., Liddle, P.F., 2012. Differential effects of surface area, gyrification and cortical thickness on voxel based morphometric deficits in schizophrenia. Neuroimage 60,693-699. doi:10.1016/j.neuroimage.2011.12.058

Raichle, M.E., MacLeod, A.M., Snyder, A.Z., Powers, W.J., Gusnard, D.A., Shulman, G.L., 2001. A default mode of brain function. Proc Natl Acad Sci U S A 98, 676-682. doi: $10.1073 /$ pnas.98.2.676

Sarvas, J., 1987. Basic mathematical and electromagnetic concepts of the biomagnetic inverse problem. Phys Med Biol 32, 11-22.

Schnitzler, A., Gross, J., 2005. Normal and pathological oscillatory communication in the brain. Nat Rev Neurosci 6, 285-296. doi:10.1038/nrn1650

Schölvinck, M.L., Leopold, D.A., Brookes, M.J., Khader, P.H., 2013. The contribution of electrophysiology to functional connectivity mapping. Neuroimage 80, 297-306. doi: $10.1016 / j$.neuroimage.2013.04.010

Smith, S.M., Fox, P.T., Miller, K.L., Glahn, D.C., Fox, P.M., Mackay, C.E., Filippini, N., Watkins, K.E., Toro, R., Laird, A.R., Beckmann, C.F., 2009. Correspondence of the brain's functional architecture during activation and rest. Proc. Natl. Acad. Sci. U. S. A. $106,13040-13045$. doi: $10.1073 /$ pnas.0905267106

Stevenson, C., Brookes, M., López, J.D., Troebinger, L., Mattout, J., Penny, W., Morris, P., Hillebrand, A., Henson, R., Barnes, G., 2014. Does function fit structure? A ground truth for non-invasive neuroimaging. Neuroimage 94, 89-95. doi: $10.1016 /$ j.neuroimage.2014.02.033

Stufflebeam, S.M., Liu, H., Sepulcre, J., Tanaka, N., Buckner, R.L., Madsen, J.R., 2011. Localization of focal epileptic discharges using functional connectivity magnetic resonance imaging. J. Neurosurg. 114, 1693-1697. doi:10.3171/2011.1.JNS10482

Tewarie, P., Bright, M.G., Hillebrand, A., Robson, S.E., Gascoyne, L.E., Morris, P.G., Meier, J., Van Mieghem, P., Brookes, M.J., 2016. Predicting haemodynamic 
networks using electrophysiology: The role of non-linear and cross-frequency interactions. Neuroimage 130, 273-292.

doi:http://dx.doi.org/10.1016/j.neuroimage.2016.01.053

Tewarie, P., Hillebrand, A., van Dellen, E., Schoonheim, M.M., Barkhof, F., Polman, C.H., Beaulieu, C., Gong, G., van Dijk, B.W., Stam, C.J., 2014. Structural degree predicts functional network connectivity: A multimodal resting-state fMRI and MEG study. Neuroimage 97, 296-307. doi:http://dx.doi.org/10.1016/j.neuroimage.2014.04.038

Troebinger, L., Lopez, J.D., Lutti, A., Bradbury, D., Bestmann, S., Barnes, G., 2014. High precision anatomy for MEG. Neuroimage 86, 583-591. doi:10.1016/j.neuroimage.2013.07.065

Tzourio-Mazoyer, N., Landeau, B., Papathanassiou, D., Crivello, F., Etard, O., Delcroix, N., Mazoyer, B., Joliot, M., 2002. Automated anatomical labeling of activations in SPM using a macroscopic anatomical parcellation of the MNI MRI single-subject brain. Neuroimage 15, 273-289. doi:10.1006/nimg.2001.0978

van Dellen, E., Douw, L., Hillebrand, A., Ris-Hilgersom, I.H.M., Schoonheim, M.M., Baayen, J.C., De Witt Hamer, P.C., Velis, D.N., Klein, M., Heimans, J.J., Stam, C.J., Reijneveld, J.C., 2012. MEG Network Differences between Low- and High-Grade Glioma Related to Epilepsy and Cognition. PLoS One 7, e50122. doi:10.1371/journal.pone.0050122

Wens, V., Bourguignon, M., Goldman, S., Marty, B., Op de Beeck, M., Clumeck, C., Mary, A., Peigneux, P., Van Bogaert, P., Brookes, M.J., De Tiège, X., 2014. Inter- and Intra-Subject Variability of Neuromagnetic Resting State Networks. Brain Topogr. 27, 620-634. doi:10.1007/s10548-014-0364-8

Wens, V., Marty, B., Mary, A., Bourguignon, M., Op de Beeck, M., Goldman, S., Van Bogaert, P., Peigneux, P., De Tiege, X., 2015. A geometric correction scheme for spatial leakage effects in MEG/EEG seed-based functional connectivity mapping. Hum Brain Mapp 36, 4604-4621. doi:10.1002/hbm.22943 